\title{
Uniqueness in the life sciences: how did the elephant get its trunk?
}

\author{
Andrew Buskell ${ }^{1}$ (D) Adrian Currie ${ }^{2}$
}

Received: 28 July 2020 / Accepted: 23 June 2021 / Published online: 19 July 2021

(c) The Author(s) 2021

\begin{abstract}
Researchers in the life sciences often make uniqueness attributions; about branching events generating new species, the developmental processes generating novel traits and the distinctive cultural selection pressures faced by hominins. Yet since uniqueness implies non-recurrence, such attributions come freighted with epistemic consequences. Drawing on the work of Aviezer Tucker, we show that a common reaction to uniqueness attributions is pessimism: both about the strength of candidate explanations as well as the ability to even generate such explanations. Looking at two case studies-elephant trunks and human teaching-we develop a more optimistic account. As we argue, uniqueness attributions are revisable claims about the availability of several different kinds of comparators. Yet even as researchers investigate the availability of such comparators, they are able to mobilize complex sets of empirical and theoretical tools. Rather than hindering scientific investigation, then, we argue that uniqueness attributions often spur the generation of a range of epistemic goods.
\end{abstract}

Keywords Uniqueness $\cdot$ Comparative Biology $\cdot$ Explanation

\section{Introduction}

Uniqueness has received little attention in recent philosophy of science. This is surprising given its important role in the life sciences. There it is often claimed that events, traits, or lineages are unique; for example, that evolutionary events are contingent (McConwell 2019, Currie 2018), irreversible (Maynard-Smith \& Szathmary, 1995) or idiosyncratic (Wong 2019); that human beings evolve under unique cultural

Andrew Buskell

abuskell@gmail.com

1 Department of History and Philosophy of Science, University of Cambridge, Free School Lane, Cambridge CB2 3RH, UK

2 Department of Sociology, Philosophy and Anthropology, University of Exeter, Amory Building, Rennes Drive, Exeter EX4 4RJ, UK 
circumstances (Henrich 2015); and that lineages bear unique, novel traits (Wagner 2014). The metaphysics of evolutionary kinds further suggests an important role for uniqueness. The dominant view understands such kinds as individuals: particular trajectories deserving of narrative explanation (Hull 1976).

In this paper, we provide an analysis of uniqueness; one that sheds light on the nature and role of uniqueness attributions in the life sciences. Though there are metaphysical issues in the neighborhood, we focus on the epistemological implications of uniqueness attributions. More specifically, our attention focuses on unique trait attributions. Unique traits are non-recurrent and as such, limit researcher abilities to acquire evidence, test hypotheses and provide explanations concerning them.

These epistemic limitations are most evident in those areas of the life sciences that deploy historical explanation. While inheriting well-known problems with historical explanation more generally, ${ }^{1}$ explanations of uniqueness-the emergence of a new species, trait, or kind of evolutionary individual-face additional sources of pessimism. After all, if a trait is non-recurrent, then it seems incomparable: other lineages won't possess it. And if this is so, one should be gloomy about the explanatory prospects of comparative approaches.

In the face of such pessimism, we point to heterogeneous means and methods for gathering evidence and providing explanations in the life sciences. These provide the foundation for a more optimistic take on the role of uniqueness attributions. We build our account by examining when evolutionary researchers make uniqueness claims and how they then investigate them. Employing two case studies-elephant trunks and human teaching-we show how scientists group together traits into contrast classes using criteria of similarity. Affordance similarity groups together traits that display qualitative similarities in the affordances they exploit, while evolutionary similarity groups together traits on the basis of similar evolutionary circumstance. As we argue, there are reasons to be optimistic whichever criterion a researcher adopts: non-recurrence does not preclude sophisticated and powerful means of evolutionary investigation and explanation.

Our analysis proceeds in two stages. In the first, we begin by zeroing in on traits as a target of uniqueness attributions in the life sciences ('The uniqueness of what?'), before characterizing the relationship between uniqueness, incomparability and pessimism ('Uniqueness and pessimism in the life sciences') and discussing Aviezer Tucker's (1998) pessimistic conclusions around uniqueness and explanation ('Tucker on uniqueness'). This stage ends with an analysis that distinguishes two broad kinds of comparison ('Pessimism and comparisons'). These form the basis for the second stage of analysis which shifts to look at how uniqueness attributions are made in biological practice. Here we introduce some conceptual machinery to make sense of the comparative methods at work in the life sciences ('Reasons for optimism: two case studies') before examining two case studies-elephant trunks ('The

\footnotetext{
1 Notably, that historical explanations provide mere 'how possibly' explanations rather than 'how actually' ones (Dray 1957, Gallie 1964, Ereshefsky \& Turner, 2019); that they provide 'explanation sketches' that lack nomological power (Hempel 1942); or that they lack confirming instances and access to crucial information (Collingwood 1936/1976).
} 
elephant's trunk') and teaching in hominins ('Human teaching')—with some stocktaking in between ('Interlude: selection regimes and evolvability'). These examples underwrite our argument for a more optimistic take on uniqueness attributions, one that we defend from potential complications ('Human teaching'). As we argue, understanding uniqueness attributions sheds light on the comparative strategies at work in the life sciences by showing how researchers wield a variety of tools to get a handle on the spectacular and peculiar outputs of the evolutionary process.

Some initial caveats. First, while we offer some examples (in 'The uniqueness of what?') demonstrating the widespread interest in uniqueness, we do not aim to provide an exhaustive survey of all the areas or topics in the life sciences where uniqueness attributions might be made. Second, and as noted above, 'uniqueness' is only infrequently an explicit target in life sciences research. This is in part because the term isn't a common one in biological nomenclature. It may also reflect the fact that researchers have developed various strategies for situating putatively unique traits in comparison classes, as we demonstrate below. Our analysis, then, is not focused on explicating the term, but instead focuses on how scientists grapple with non-recurrent events, employ strategies and tools to make sense of them, and justify the explanations they give.

This analytical strategy comes with potential limitations. The epistemic problems of uniqueness we discuss may depend on the particular investigative aims and methods at hand. In this paper, we focus on the methods of comparative biology. Nonetheless, we suspect our account of uniqueness attributions carries over into other areas of the life sciences. We also suspect that the broadly optimistic stance towards uniqueness attributions can be extended beyond the life sciences. We leave these broader explorations, however, for later work.

\section{The uniqueness of what?}

When researchers in the life sciences claim that a creature is unique, what do they mean? Though one might make such claims in terms of 'events', 'processes', or 'lineages', we will understand such claims to be about 'trait' uniqueness. To see why, consider a puzzle that arises when such claims are situated in the context of 'lineages'.

At a first pass, calling a lineage 'unique' seems either trivial or confused. According to evolutionary systematics, every lineage is the result of a unique branching event. And yet at the same time, every lineage traces its ancestry to a common ancestor. Does this mean that all lineages are unique? That no known lineages are? If the former, uniqueness attributions are uninformative, simply restating results from systematics; if the latter, such attributions seem spooky, suggesting unique events fall outside the remit of contemporary scientific understanding. This sets up a challenge for any account of biological uniqueness: identify notions which are neither trivial or uninformative, nor confused or spooky.

Happily, shifting the context of uniqueness attributions from lineages to traits answers the challenge. One can understand a trait's uniqueness in terms of comparisons indexed to particular descriptions. Are elephant trunks unique? This depends 
on, first, how one describes the trait at hand. As we'll see, described as proboscises - an evolutionary development of the mammalian snout—-trunks may be compared to the tapir's flexible snout. But given more specific descriptions, trunks may belong to elephants alone. Second, uniqueness depends on how one situates the trait phylogenetically. Trunks are not unique to Loxodonta africana: they are also possessed by forest elephants in African and Indian elephants (as well as various extinct Elephantidae). And so asking whether trunks are unique requires the right phylogenetic scope. Nonetheless, unique trait attributions are neither trivial nor uninformative: after fixing descriptors and comparators, uniqueness is an empirical question and-as we'll see in the next section-one with epistemic upshots. ${ }^{2}$

Interestingly, we think understanding uniqueness attributions as being about traits allows one to understand similar claims made in terms of lineages, events, or processes; a lineage is unique just in case it possesses a unique trait (mutatis mutandis for events and processes).

In addition to clearing up conceptual issues, understanding uniqueness attributions in this way has empirical benefits, allowing for fruitful connections to work on evolutionary novelty and innovation in the evolutionary developmental biology literature. Nonetheless it is worth distinguishing our target and approach from this neighboring literature on two counts. First, 'novelty' and 'trait-uniqueness' need not overlap. The elephant's proboscis is likely a novelty in that lineage, but it does not follow from this that it is unique if tapirs possess them as well. Second, philosophical discussion of novelty has focused on explicating the concept and its role in evolutionary theory (Pigliucci 2008; Brigandt \& Love, 2010, Wagner 2014). By contrast, we are interested in the epistemic consequences of uniqueness attributions and how they shape research in the life sciences more generally. While we suspect that useful connections between our arguments and that literature may be drawn, we leave drawing these for later work.

\section{Uniqueness and pessimism in the life sciences}

When researchers make uniqueness attributions their claims are decidedly not vapid or spooky: they take these attributions to have important epistemological consequences. Consider an illustrative example. Smith and Wood, (2017) argue that unique hominin traits block epistemic access to the evolutionary past. They begin by defining uniqueness:

\footnotetext{
2 It does not follow that all uniqueness attributions will be compelling: defining a trait in an extremely fine-grained or coarse-grained manner will trade off against pragmatic demands and empirical fecundity. This means that while uniqueness attributions are relative to the interests of researchers, this is epistemically benign. As we describe in more detail below, scientists investigate putatively unique traits at multiple levels of description. This is a deliberate strategy to identify areas of epistemic traction. Yet whether or not a trait is unique at any particular level of description is not settled in advance, but is a revisable claim subject to empirical investigation.
} 
We define a feature or trait to be "unique" when an investigator concludes that there are no comparative data against which the features can be evaluated, interpreted, or contrasted... By definition, just as an historical event is blocked from experimental evaluation, a unique feature, trait or event is blocked from direct comparative evaluation (673).

Smith and Wood connect uniqueness attributions to the prevailing comparative methods in the life sciences. Can the trait be juxtaposed with other traits? Is hominin tool use, say, meaningfully comparable with tool use in corvids? ${ }^{3}$ Or in in our fellow primates? Smith and Wood argue that many hominin traits have no true comparisons and this in turn limits what we can learn about them-stressing the vexing situation of hominins and their distinctive complex of novel traits. ${ }^{4}$ The thought is that because one cannot set traits in a comparative context, one thus lacks epistemic access to them.

[researchers are tempted] to pose and try to answer, tempting but unrealistic research questions. There is much we would like to know about human evolutionary history, but wanting to know something does not make it knowable (ibid, 677).

We take Smith and Wood to be giving a particularly clear example of a fairly widespread position in the life sciences: that because a trait is unique, it cannot be placed in a comparative context and thus one lacks the resources available to generate robust scientific knowledge. Similar logic underwrites the "N=1" problem in astrobiology (Powell 2020) and the discussions around 'singletons' in macroevolutionary contingency (Vermeij 2006; Losos 2017; Wong 2020). In such cases, the non-recurrence of some trait (tool-use, carbon-based life, distinctive Dinosaurian traits) underwrites pessimism about epistemic access and explicability. We think this epistemic pessimism is widespread in the life sciences-even if, as we noted above, researchers do not often use the term 'unique' for various nomenclatural reasons.

The discussions of this and the previous section provides the orientation for the remainder of our paper. We're interested in unique trait attributions; substantive empirical claims that are neither vapid nor non-naturalistic. Moreover, such attributions have epistemological consequences: unique targets are problematic because they are non-recurrent and non-recurrent events cannot be put in comparative context. Lastly, researchers across the life sciences often adopt a pessimistic epistemic stance, questioning whether epistemic goods can be generated from a study of nonrecurrent traits.

\footnotetext{
3 We have used 'hominin' throughout this paper to refer to the group of species consisting of modern humans and their immediate ancestors following the split from Pan. Prior to recent changes in taxonomic nomenclature, 'hominid' was used with the same extension. Quotes below that employ 'hominid' should be understood in this way.

${ }^{4}$ Human uniqueness is most often discussed in the philosophical literature in terms of a 'human nature'. For recent work surveying this topic, see: Kronfeldner (2018) and the essays in Hannon and Lewens, (2018). For a recent historical account that charts twentieth-century claims of human uniqueness, see: Milam (2019)
} 


\section{Tucker on uniqueness}

What is the problem of non-recurrence? Our jumping off-point looks to the work of Tucker (1998), who defines something as unique when it lacks comparisons and thus (so he claims) lacks what is needed for justified scientific understanding. Although he focuses generally on 'events', the account generalizes to the study of any nonrecurrent entity, like traits: ${ }^{5}$

"There are no acceptable scientific theories that can explain unique events because unique events: Occur once and only once. Their significant properties or parameters, specified in the topic description of the why-question, are either:

a. Not shared by any other event, apart from spatiotemporal location and self identity (or it is unknowable whether they are shared by other events). Or

b. Too complex or chaotic for effective comparison with other events." (Tucker 1998, 65)

This calls for unpacking. Tucker employs van Fraassen's pragmatic account of explanation (1980). This takes explanations as answers to why-questions. These have topic-descriptions that highlight aspects (properties, parameters) of the target to be explained. To anticipate later discussions, one example might be the question 'why are elephant trunks such remarkably flexible, multi-use organs?' In answering this question, aspects of elephant behaviour and anatomy would be highlighted, perhaps behaviours such as the trunk 'hand-shake' greeting. By contrast, a question such as 'why did elephant trunks evolve?' would highlight aspects of elephant evolutionary history, properties of elephant ancestors and the environment in which these populations evolved. Thus, one upshot of Tucker's view (which we fully endorse) is that uniqueness attributions are sensitive to description. As we've argued, a trait's uniqueness depends in part on how it is described. The elephant's trunk might be unique vis-à-vis proboscises, but it certainly isn't unique vis-à-vis having nostrils.

Tucker's analysis highlights two ways in which an event can be incomparable. The first occurs when some features are only tokened by the event in question; such features are theirs alone. Elephant trunks (properly described) might be held by elephants and elephants alone. Alternatively, comparisons may be lacking because the inductively salient features of events are inaccessible. On the former, events are (so far as can be known) non-recurrent; on the latter, there may be comparable events,

\footnotetext{
${ }^{5}$ Does Tucker's argument generalize? A concerned referee worried that Tucker's "events" might not apply to "traits". We think it does so straightforwardly. First, occurrences of traits are a kind of event. Unless there is a distinction to be drawn between 'events' generally and 'trait-events' specifically—something we are skeptical of - this worry does not get off the ground. Second, Tucker's analysis doesn't turn on talk of events, processes, or traits, but rather on circumstances where investigative targets lack comparators. And as we have shown, researchers in the life sciences take unique traits to be such targets.
} 
but we lack the means (because of epistemic or practical limitations) to discern them.

Tucker's account is neither vapid nor spooky. And he is clear on the epistemic consequences of uniqueness: it rules out scientific explanation. ${ }^{6}$ The equivalence of uniqueness with incomparability and the corresponding pessimistic conclusion raise questions as to how researchers in the life sciences could ever investigate putatively unique events. Yet researchers $d o$ investigate such events - and seem to be successful at doing so.

\section{Pessimism and comparisons}

Tucker, we think, gets a lot right about uniqueness: uniqueness is sensitive to description and the epistemic limitations of uniqueness result from difficulties in drawing comparisons. Still, we think that Tucker's stance is too pessimistic. His analysis shifts too quickly from noting incomparability (in his sense) to the impossibility of a scientific work on unique events.

So what counts as a comparison? We think Tucker's appeal to the concept is ambiguous. Borrowing from Currie (2018), let's consider two kinds of comparisons. The first identifies comparisons by tracing causal pathways. Elephants, for instance, interact with their environment: knocking down trees, feeding, migrating and so forth. These causal connections can empirically inform researchers about the critter at hand: trees broken in this way and at this height are evidence of recent elephant activity. Such causal pathways can be traced at greater or lesser spatiotemporal scales. Specific elephants might have idiosyncratic ways of clearing tracks and these can serve as signals for their individual activity. More zoomed out, the way that different elephant populations cut a trail can be very different. Zoomed out even further, one can compare how elephants are related to each other and to other lineages via causal lines of ancestry-homologies-which may be used to underwrite comparisons between and within taxa. Here researchers compare states of the target itself at different times; sometimes directly, while at other times, as inferred by their effects on the world. The second kind identifies comparisons with tokens of some type. Here, although there is no relevant causal chain linking comparisons, they count as such in virtue of being instances of the same type of event or process. If both elephants and tapirs have proboscises and these evolved (more or less) independently, then one has two instances of the evolution of the proboscis type. Together these instances can underwrite generalizations about the evolution of the trait.

These two kinds of comparisons generate different epistemic goods. Causal-pathway comparisons provide information about particular features of the target while event-type comparisons furnish information about regularities; causal-pathway

\footnotetext{
${ }^{6}$ Tucker discusses several strategies that scientists adopt in light of apparent uniqueness, emphasizing in particular mechanistic strategies which might reveal underlying commonalities after all. We welcome this move and note that it shares similarities with the strategy we adopt below. However, unlike Tucker, we take this strategy to undercut the equivalence between uniqueness and incomparability.
} 
comparisons illuminate by tracing causal consequences back to features of the target while event-type comparisons evidence generalizations (laws, models) applicable to the target. Particular instances of evidence might not neatly fall exclusively into one of these categories - comparisons will often play both roles-but the distinction is important and plays a key role in our positive account.

Tucker's definition of uniqueness relies solely on event-type comparisons. Although Tucker is rightly dismissive of Deductive-Nomological accounts of explanation, he nonetheless retains the idea that explanation requires some kind of modal force-one needs to know how the target may have behaved differently-and to do so one needs comparisons capable of informing generalizations. As such, he requires that explanations involve generalized models of dynamics and behaviour.

by definition, [unique] events exclude the possibility of other events that can be relevant for confirming the theoretical backgrounds that can participate in their explanation because: Unique events are not recurrent; their significant properties are either not shared by any other event or it is impossible to know whether other events share them; or they are too chaotic and complex for effective comparison with other events. Explanations of unique events are radically underdetermined because any theoretical background that is relevant for their explanation is underdetermined. (Tucker 1998, 66)

'Theoretical backgrounds' provide the modal force required to understand how the target may have turned out differently under counterfactual circumstances-and comparisons are required to establish these backgrounds. On our reading of Tucker, something is unique just when it lacks event-type comparisons. ${ }^{7}$

Tucker's account is epistemically pessimistic in an important sense, but noncommittal in another. Unique events are beyond the reach of acceptable scientific explanation because such explanations require the application of justified models of event regularities. Yet these can only be achieved by examining relevantly similar events and without any such events, regularities cannot be identified or confirmed. $\mathrm{He}$ is, however, non-committal about when and if any particular targets are in fact unique in his sense: the extension of 'uniqueness' might be empty. As we'll see, our disagreement with Tucker, then, turns on what counts as a comparator and what is required to provide an 'acceptable scientific theory'.

This pessimism extends to the generation of explanations. Tucker suggests that unique events generate explanatory underdetermination: researchers lack sufficient comparisons to empirically support the regularities underwriting our explanations. ${ }^{8}$ Smith and Wood's pessimism too can be understood along these lines. They deny

\footnotetext{
7 We note that both 'uniqueness' and 'non-recurrence' can come in degrees, depending on how finely specified the relevant target is and how much 'wiggle-room' there is for something to count as a comparator. At some level of description, the mass extinction event 66 million years was unique and non-recurrent. But at another level of description it is part of a set of five (perhaps soon six!) mass-extinctions: it is recurrent.

8 This kind of underdetermination is distinct from more familiar notions where researchers lack sufficient evidence to distinguish between competing hypotheses (Turner 2004; Tucker 2011; Currie 2018 chapter 4).
} 
that researchers can gain empirical traction on hominin evolution because, on their view, hominins are the bearers of multiple unique traits that block researchers from marshalling comparisons. Where Tucker makes a conceptual point about the epistemic consequences of uniqueness, Smith and Wood argue that hominin traits are in fact unique and thus not amenable to scientific discovery or explanation.

We think this pessimism moves to far too fast. There is, we suggest, plenty of room for more optimistic takes. At least within the life sciences, researchers leverage causal-pathway comparisons to generate knowledge about the salient properties, mechanisms and processes at work in unique events. And overlapping with these are coarse-grained event-type comparisons that can capture and explain inductively relevant clusters. Together, these strategies can yield complex, highly tailored explanations of target events.

\section{Reasons for optimism: two case studies}

Scientists are skilled at finding causal regularities in the world, leveraging and integrating both causal-pathway and event-type comparisons. The regularities they discover are defeasible but inductively salient. In general, researchers adopt various criteria of similarity in order to import tools, models and methods from elsewhere in evolutionary biology and beyond. Such criteria of similarity help identify clusters of properties presumed to be inductively salient. These presumptive clusters can then create suitable contrast classes against which hypotheses, models and explanations can be tested.

As we demonstrate below, life scientists use similarity criteria to construct comparison classes and apply tools, methods and regularities to putatively unique traits. We highlight two such criteria here: affordance similarity and evolutionary similarity. Both guide researchers in the construction of contrast classes in different ways. Affordance similarity highlights similarities between organismic behavioural repertoires and features in the world; evolutionary similarity the extent to which organismic traits are presumed to be similar and subject to similar evolutionary processes. No doubt there will be other kinds of similarity that biologists focus on-the complexity and messiness of life begets a complex and messy set of scientific approaches and categories-but nonetheless affordance and evolutionary similarity are critical notions for understanding how scientists tackle putative uniqueness in the comparative cases we're focused on.

Importantly, we further break down evolutionary similarity into two distinct criteria, each of which furnishes a characterization of uniqueness. The first, similarity in selection regimes highlights how shared selection pressures can generate similar traits. On this criterion, uniqueness is equated with being a statistical outlier: traits at an extreme end of a statistical distribution that result from severe and/or persistent selection regimes. The second, evolvability similarity, groups together traits on the basis of dispositional tendencies of lineages to evolve in similar ways. Here, unique traits are the result of path-dependent cascades. Briefly, one can understand a 'path-dependent cascade' using machinery developed in the contingency literature. Desjardins (2011a, b) defines path dependence as holding when an outcome is 
probabilistically dependent on the occurrence (or non-occurrence) of specific events along a causal pathway, which he distinguishes from dependence on initial conditions. For the latter, where one begins matters, for the former, what happens along the way matters. A trait is the result of a path-dependent cascade when an evolutionary trajectory diverges from relevant contrasts in exploring a different region of evolutionary space as a result of specific events along its evolutionary pathway.

We develop and clarify much of this machinery in our discussion of the elephant's trunk. As we conclude, whether the trunk is best understood as unique in the sense of being a statistical outlier or a path-dependent cascade is still very much up for grabs in the empirical literature. We then turn to human teaching. Here we suggest that accounts attempting to characterize human beings as mere statistical outliers is on shaky ground; there seems to be increasing evidence that human teaching-as well as several other capacities - is most fruitfully understood as the outcome of a path-dependent cascade.

\section{The elephant's trunk}

Elephants are remarkable. Originating approximately forty million years ago, the lineage quickly evolved the basic pachyderm model: large statures, big brains and, of course, trunks.

Trunks are powerful and dextrous. They can be used to lift logs over 300kgs as well as to "perform amazingly delicate functions, such as picking up a peanut, cracking it open with the tip on the back side of the trunk, blowing the shell away and eating the kernel" (Shoshani 1998, 487). Not used solely to manipulate objects, elephants also employ their trunks to 'snorkel' while submerged; to perform social functions such as a 'trunk-shake' greeting; and to manufacture tools. Are trunks unique? Answering this question requires both describing trunks and situating them in comparative contexts.

Often, scientists leverage similarity in affordances to group together similar creatures. Originating with Gibson (2015/1986), affordances are what the environment "offers the animal, what it provides or furnishes, either for good or ill" (Gibson 2015/1986, 119). Affordances involve a relation between an organism and specific features of its environments: the media in which it survives, the surfaces with which it interacts and the active behaviour of other goal-directed critters (ibid. 122-127). Building on the work of contemporary ecological psychologists, we understand affordances broadly as 'opportunities for behaviour': suites of relationships constituted by repertoires of possible organismic behaviours and features of the world exploited by the organism in pursuit of goals. (Chemero 2009, 151; Walsh 2015). Affordances, then, are relationships between an organismic relatum (a behavioural and phenotypic repertoire) and an environmental relatum (the set of features exploitable by such behaviours). ${ }^{9}$ On our view, there are often constructive relationships

\footnotetext{
9 Affordances depend on traits. Without delving too deeply into metaphysical issues, one can think of them, along with features, as INUS conditions for realizing affordances (Mackie 1965): specific traits and features are insufficient but non-redundant parts of an unnecessary but sufficient condition for exploiting something as, say, graspable or liftable or edible. Construing affordances in this way is meant to capture the idea that an organism's behavioural repertoire might support a wide range of affordances, but does
} 
between these relata: just as organisms are shaped by their environment, so too are environments actively shaped by organisms.

How might this strategy apply to the trunk? Consider Milewski and Dierenfeld, (2013), who group the elephant trunk into a broad trait category they call proboscises: "flexible, tubular extension of the joint narial and upper labial musculature that is, at least in part, used to grasp food" (85). This identifies similarities as a specific kind of affordance (grasping) associated with a specific morphological structure (roughly, snouts). So understood, elephants are not alone in having a proboscis. Tapirs have them too. Like elephants, tapir proboscises are flexible, tubular narial projections used to grasp food. Nonetheless, there are significant differences in the extent to which their proboscises facilitate grasping.

Elephant trunks can grasp and manipulate objects with two distinct kinds of opposable grips. The first involves the familiar curling of the trunk around objects. This uses the ventral side of a trunk to create an opposable surface, akin to the way that fingers curl around the handles of mugs. The second kind of grasping is more striking. Elephant trunks have enlarged projections called 'trunk tip fingers', which they can use to carry out fine-grained grasping and manipulation like nut-cracking and paintbrush-holding (Rasmussen and Munger, 1996). ${ }^{10}$ Contrast these capabilities with those of tapirs. Their proboscises are more akin to a flexible shovel: able to move food toward the mouth, but lacking opposability and thus with only minimal affordances for true grasping. So, although both tapir and elephants have some similarities in affordances, the elephant trunk is nonetheless distinctive.

Many researchers employ affordances as a means of crafting hypotheses about evolutionary history; similar affordances may be the result of similar evolutionary circumstances. But how one locates that similarity generates different kinds of uniqueness attributions and different kinds of explanations and inferences.

Let's compare two evolutionary narratives concerning elephant trunks.

The first narrative links the evolution of the trunk to a common adaptive trend among large mammalian herbivores; namely, the expansion of soft mouth parts (Pretorius et al. 2016). As herbivores increase in size, they need to consume more food. Yet while bigger mouths afford bigger bites in absolute terms, the relative bite size and quality of these bites decreases: for both browsers and grazers, larger bites risk consuming more of the fibrous parts of plants (stems and twigs) and less of the nutritive parts (leaves). As a result of this, large mammalian herbivores have evolved a range of strategies for boosting feed quality by enlarging their soft mouth parts (lips, tongues, narial extensions). These increase the quantity of food per bite while providing the means for fine-grained selection of foodstuffs. The moose's prehensile upper lip, for instance, allows them to strip leaves from branches. A similar version

\section{Footnote 9 (continued)}

not do so invariably, because specific features might not (or may no longer) be found in the critter's environment. An elephant's trunk can lift objects that weigh in excess of $300 \mathrm{~kg}$-but only if there are such objects in the environment to be lifted.

${ }^{10}$ Asian elephants (Elephas maximus) have a single, dorsal projection, whereas African elephants (Loxodonta africana and cyclotis) have both a dorsal projection and a ventral projection. 
of this strategy is the long, prehensile tongue of giraffes. Perhaps the trunk is simply an exaggerated outcome of similar selection pressures.

On this narrative, the trunk's evolution is an extreme version of a more general evolutionary trend, resulting from a scaling relationship among body size, bite size, bite quality and length of soft mouth parts; trunks provide similar sets of affordances to giraffe tongues and moose lips. Nonetheless, the trunk is still unique in a sense: it is a statistical outlier. The statistical pressures that operates on large mammals were merely stronger, or operated for longer, on the elephant. To get to an elephant's trunk, one simply needs to begin with a large mammal — a tapir, say — and 'turn evolution's crank': sufficient selection pressure for increased mouth parts opens the path to trunks.

Let's turn to an alternate account. On this second evolutionary narrative, elephants evolved in a strikingly divergent way from other ungulates. This narrative begins by situating proto-elephants in aquatic or semi-aquatic environments. This isn't so outlandish as it may seem, as some of the elephant's closest living relatives are the sea-cows (manatees and dugongs). On this 'aquatic elephant' hypothesis, what drove trunk evolution was not grasping food but snorkelling: in the aquatic environments in which they found themselves, it was adaptive to move submerged through water. It was only later that the trunk was co-opted for increased grasping functionality.

On this account, there is no easy route from a tapir-like trunk to an elephant's trunk: the evolutionary path leading to elephant trunks was shaped by a distinct set of selective pressures that required not only specific starting conditions to get off the ground, but also further events downstream. By this hypothesis, both specific proto-elephant traits and a semi- or fully-aquatic environment, along with the later co-option of these traits in a terrestrial niche, were required to evolve the highly plastic, multi-purpose organ. If this latter account is right, then although one might class both elephants and other mammals together, one nonetheless cannot use tapirs (or other large ungulates) as models for the elephant's evolution, nor can one take the elephant as the extreme end of an evolutionary trajectory that tapirs are potentially traversing. This is because, on this account, trunks are the outcome of a pathdependent cascade. Trunk evolution was dependent on multiple events: elephants having particular morphology, being located in (semi-) aquatic environments and so on.

If something like the first narrative is right, then elephant trunks are unique not because of the kind of selection pressures they encountered, but because the persistence and strength of selection pressures generated a trait at the extreme end of a statistical distribution. Being unique in this sense does not warrant pessimism: there are bountiful event-type comparisons to other mammals that underwent similar evolutionary journeys. If the latter narrative is right, then there are no event-type comparisons: no other lineage has pursued the same evolutionary trajectory. This might warrant some pessimism, for reasons discussed by Tucker above. Nonetheless, one is able to narrow down possible causes by using causal-pathway comparisons: by looking at phylogenetic neighbours and by employing rich sources of paleobotanic, paleoclimatic and paleontological evidence. So even here, there is reason to be optimistic. 


\section{Interlude: selection regimes and evolvability}

There are a number of lessons that can be drawn from this case study. First, it is a common strategy to lump together entities on the basis of inductive similarity. And as the above shows, researchers have a wide range of tools for lumping together putatively unique events into groups with similar enough comparators to warrant the application of more general causal regularities. One way of lumping employs causal-pathway comparisons. In this regard and despite their uniqueness, elephant researchers are in an enviable epistemic situation as compared to those studying other lineages:

Unlike researchers who study dinosaurs and have to rely on distant living relatives, such as crocodiles and birds for indirect comparison, students of Proboscidea are fortunate to have living models with which to compare and contrast the extinct taxa directly. This advantage should not be underestimated, because knowledge of the living species helps one to interpret observations of extinct forms and vice versa (Shoshani et al. 1998, 487)

Shoshani's point is that those studying some extinct lineages (dinosaurs, for instance) have problematic causal-pathways comparisons-birds and crocodilians are problematically divergent from dinosaurs-but Proboscidea have bountiful such comparisons: namely, currently existing species. So even to the extent that elephant researchers lack event-type comparisons (more on this below), there are bountiful causal-pathway comparisons. This rich information allows researchers explore the range of variation in relevant contrast classes, provides statistical access to models of their behaviour, physiology and so forth and allows for the testing of evolutionary narratives. One can even identify homologous relationships between extant and extinct elephants and draw on parallel evolution within the clade.

A second set of lessons comes from noting some of the differences in epistemic strategy - and epistemic consequences - that come from applying one or the other of the evolutionary similarity criteria. Rather than focus on providing a fully detailed picture of the morphology and affordances of the trunk (underpinning, for instance, snorkelling, nut-cracking and social greetings) Milewski and Dierenfeld, (2013) use affordance-similarity to motivate an evolutionary narrative that unifies elephants, tapirs and other extinct proboscis-wielding critters. This narrative moves from a causal-pathway comparison-based on similarity in ecological circumstance- to an event-type comparison based around similar adaptive strategy. As we showed, Pretorius et al. claim that the elephant can be fruitfully compared to other large-bodied mammalian herbivores: the elephant trunk can be situated along a distribution of adaptively similar traits that link body size, relative availability and quality of food and the size of soft mouth parts. ${ }^{11}$

\footnotetext{
11 There is a rich epistemic discussion to be had concerning how satisfying we should find explanations like Pretorius et al.'s: under what conditions should we find explanations at such extremely abstract levels of detail, when applied to complex and putatively unique traits acceptable? For better or worse, we leave this discussion for later work.
} 
Two points are worth making regarding this strategy. First, although these moves generate explanations by unifying different species into a shared (if abstract) evolutionary trajectory or set of affordances, the unity at work is not a broad 'super-empirical' virtue that one might use to decide between competing explanations (Churchland 1985; Kitcher 1981, 1989). Instead, the strategy attempts to highlight deep causal regularities between evolving systems and environments, with abstraction facilitating the identification and evidencing of such regularities (Strevens 2008). Second, the two evolutionary similarity criteria identify different kinds of regularities and thus establish different kinds of uniqueness attributions. They do so in part because the contrast classes are established using different kinds of similarity.

This can be seen by comparing Pretorius et. al's narrative to the 'aquatic elephant' hypothesis. This latter hypothesis groups together comparators on the basis of presumed similarity in evolvability - the complex dispositional properties of lineages to explore evolutionary space (Brown 2014) — with phylogenetic proximity often serving as a proxy measure. On this account, uniqueness attributions point to specific evolutionary events as explanations for why an evolutionary trajectorydiverging from those of its comparators-could result in the trait in question. Such divergences are by their nature highly path-dependent and can involve coordinated, multi-factorial cascades of changes.

When it comes to the aquatic elephant hypothesis, one can't just turn the crankincreasing the magnitude of the same kind of selection pressure-and expect to get an elephant trunk from the tapir-like ancestor. Specific, contingent and pathdependent relationships among resource distribution, geography and phylogeny are required. Yet when it comes to the 'soft-mouth-part-hypothesis' one can just turn the crank: as ungulate herbivores increase in size, their comparative bite size decreases, as does the food quality per bite. Increasing the size of the soft mouth part mitigates both of these costs. Under these latter conditions, elephant trunks would not be divergent - they are just what happens when one turns the crank long enough. This suggests two upshots: affordance uniqueness does not entail non-recurrence and as a result, affordance uniqueness does not imply a lack of event-type comparisons (as they can be unified with others by being a statistical outlier).

Whether the elephant trunk is best explained as a statistical outlier or the result of a path-dependent cascade is up for grabs. We know of no work that contrasts the two as candidate evolutionary hypotheses. It might even be that components of the two explanations might be needed: it could be that the elephant trunk was initially enlarged as a response to the increasing size of elephant frames, only later to develop along an idiosyncratic evolutionary trajectory.

But let us turn now to consider how our account fares when dealing with a putatively unique trait in a paradigmatically unique lineage: teaching in human beings.

\section{Human teaching}

There is no doubt that Homo sapiens have a range of unique traits. The bipedal gait of humans allows their hands to be free for dextrous manipulations; the human larynx and cranial morphology supports the production of complex and 
fine-grained vocalizations; and their cognitive machinery supports abilities to produce complex, multi-step tools. For all comparative psychologists have identified similar traits in other lineages, no other critter uses their traits in such a flexible, plastic way (Premack 2010). Indeed, many human affordances result from a highly plastic cognitive system together with capacities for culture, which have supported the emergence of a bewildering range of norms, practices and technologies which enlarge both human behavioural repertoires and the features to which humans are sensitive.

A central trait supporting the transmission and proliferation of such cultural traditions is the human capacity for teaching. The capacity for highly intentional, guided, information and skill transfer turbo-charged cultural evolution by amplifying the reliance on conspecifics for acquiring sufficient resources, producing the requisite technologies and passing along specialized skills (Sterelny 2012; Tomasello 2014; Henrich 2015). On these accounts, the human capacity for teaching is key to explaining the evolutionary success and persistence of hominins, as well as the extraordinary cultural diversity our species exhibits.

We saw above Smith and Wood's pessimism about empirical access to the evolutionary history of hominins. As we suggested, this pessimism is about the lack of event-type comparisons. But we have since argued that affordance uniqueness does not entail the lack of event-type comparisons-nor that a lack of event-type comparisons hinders the application of tools, the collection of evidence and the furnishing of hypotheses that make use of causal-pathway explanations. So, how then should we understand teaching's evolutionarily uniqueness? As with the elephant trunk, there are two broad strategies by which researchers attempt to understand the putative uniqueness of human teaching.

The first strategy trades on the idea that humans are statistical outliers of a more general evolutionary trend. As we saw with elephants, such accounts lump together lineages on the basis of a presumptive cluster of projective properties. Here, the lumping occurs on the back of a broad functional characterisation of teaching. For instance, Hoppitt et al. (2008; cf. Caro and Hauser, 1992) define teaching as: “... any behaviour that is adapted to the purpose of transmitting knowledge and skills to other individuals, regardless of the proximate mechanisms involved" (489). On this account, the behaviours and capacities gathered together under the label 'teaching' represent a suite of solutions to adaptive problems that different species solve in more or less efficient manners. Humans, on this understanding, merely outperform other species:

I anticipate human teaching is at the extreme end of the distribution of teaching and that, like other apparent exceptions that in fact prove the rule, explaining teaching in humans will inform our understanding of teaching across species. (Kline 2015, 56)

These accounts adopt a coarse-grained functional schematic: if one animal (the tutor) modifies its behaviour when in the presence of another (the pupil) in such a way that (i) no benefit is gained by the tutor; (ii) the likelihood of the pupil adopting the behaviour increases, then the tutor is teaching. With this in hand, researchers are able to deduce the kinds of selection pressures that might produce teaching and as 
a consequence, to apply similar kinds of models and reasoning across the contrast class.

On this functional notion, meerkat teaching shows up as being surprisingly similar to human teaching; scorpion hunting being the prime example. Meerkat 'helpers' provision their young with scorpions in distinct stages-dead, stingless and fully functional-in a way that is indexed to the learner's age (Thornton and McAuliffe, 2006; 2008). This allows the inexperienced to learn the subtle art of scorpion-dispatching in stages. Such teaching fits the functional schematic: if one wants to eat a scorpion, biting off its stinger and passing it to a young meerkat is not beneficial to the helper (the first requirement) and a slow, staged introduction to the dangerous business certainly increases the chances of the novice to learn how to perform it (the second requirement).

Hoppitt et al. (2008) use the functional schematic to argue for a general model of teaching evolution. They focus on the role of 'inadvertent social learning' basically, the ability to learn through proximity and salience cues made or left by conspecifics-arguing that selective regimes could create an advantage for tutors to facilitate the leaving of cues and index these in ways that facilitate successful learning. This seems plausible. As meerkats are extremely cooperative, with mutualalarm signalling and alloparenting, having reliable and well-fed guards would be advantageous. Hoppitt et al. further suggest that such selective regimes are likely to come about when mere inadvertent social learning is ineffective for learning the behaviour. Here too the meerkat case fits. Inadvertent social learning might allow naïve meerkats to identify scorpions as a salient food resource, but just this piece of information is likely to be insufficient for the acquisition of requisite skills, since trial and error learning is a terrible way of learning to hunt venomous scorpions. The claim, then, is that if a particular behaviour on the part of the tutor creates conditions that encourage the successful learning of the requisite behaviour on the part of the learner, this will be selected for.

Hoppitt et al. then apply this model to hominin evolution. Unlike other Panin offshoots - say, the lineage leading to contemporary chimpanzees-they argue that hominin ancestors encountered situations like the meerkat: a wide range of scenarios where there is selective advantage to transmitting abilities and where individual trial-and-error learning is ineffective. As they suggest:

Unlike other apes, in humans, teaching could have been favoured by the requirement to transmit complicated skills and technology that are not easily acquired through inadvertent social learning. (Hoppitt et al., 491)

On both Hoppitt et al. and Kline's account, one can just 'turn the crank'; leverage a selective regime until an extreme instance is produced. Yet as with the elephant trunk, there is an alternative story which sees human teaching as the result of a very specific and path dependent cascade. Telling this story involves affirming the uniqueness of teaching in humans. As we saw with elephants, the account begins by emphasizing the affordance-uniqueness of teaching in humans and then shifts to an idiosyncratic evolutionary cascade to explain it. 
Teaching in humans involves unique affordances. ${ }^{12}$ Notably, it requires sophisticated abilities for following the intentions of others. This is fancy cognitive machinery for teachers and students alike. Teachers modify their behaviour with an awareness of the pupil's attention, slowing down and repeating some movements, correcting the pupil and so forth. Moreover, pupils must be aware that the teacher is teaching, that their behaviours are intended to communicate information: they must recognise that some of the teacher's movements are idiosyncratic, or slowed down and so forth and that these aspects of behaviour are not to be directly mimicked. In addition to the sophisticated machinery underwriting such intentional cognition, teaching requires affective components - an internal motivation to not only tolerate, but help, learners - as well as strategies for reducing the complexity of the task environment and highlighting salient features of the task for the learner.

An alternative account of the evolution of teaching takes its unique affordances to be the result of a distinct path-dependent cascade (analogous to the aquatic elephant hypothesis). This account links fluctuating climatic conditions with an already highly social species. When environments change too quickly for genetic adaptation to keep up — but not so fast as to make any attempt to learn about the world fruitless-populations can come to rely on other agents as guides to successful action (Boyd and Richerson, 1985; Shettleworth 2012). The initial tweaks that might have facilitated such a reaction may have been small: an increase in working memory, social tolerance (especially of infants) and attentive and orientation mechanisms tuned to conspecific behaviour (Tomasello 2014; Heyes 2018). But once attuned to other agents as a valuable source of getting by, however, powerful feedback loops could ratchet up the sophistication and power of such social learning (Sterelny 2012).

Teaching fits into this narrative as a fidelity ratchet; increasing the likelihood that pupils could acquire similar-enough skills, behaviours and norms to those of their tutors. Such high-fidelity transmission might become necessary when toolmaking was increasingly produced through systems of distributed labour, with different individuals responsible for making different component parts of tools, or sequential steps on the same tool (Birch 2021). Decomposing production into distinct tasks requires concepts for keeping track of distinct production roles and the ability to internalize standards of correct performance. There is good reason to think that teaching is required especially when the skills are complex and agents have only partial knowledge about how their actions lead to a final product. Under such situations, teachers would have needed to guide learners in the acquisition of abilities and to inculcate standards for producing requisite behaviour. Steps in manufacturing would need to be standardized and standards would need to be instituted and maintained by other agents.

Here we have an account - one with a broad base of evidence from archaeology, paleoanthropology, primatology and psychology—that suggests that one cannot just

\footnotetext{
12 The affordances we describe here are what the literature refers to as 'social affordances' (e.g. Rietveld 2012); opportunities for behavior solicited by the social and socially-inflected aspects of the environment.
} 
take a meerkat and 'turn the crank' to get human-like teaching. Instead, the evidence points to distinctive features of hominin evolvability: that they were highly social, tool-using, cooperative and increasingly reliant on skilled labour. In other words, hominin capacities for teaching appear to be best explored as a path-dependent cascade that sets them apart from their phylogenetic neighbours. As with the elephant case, we see two distinct investigative strategies adopted in light of the putative uniqueness of teaching. We also see that a lack of type-comparisons is no block to epistemic progress.

\section{Problems and extensions}

Let us take stock. We have identified a pessimistic epistemological stance towards explanations of putatively unique traits. Looking at the philosophical work of Tucker and appeals to uniqueness among scientists like Smith and Wood, we diagnosed this pessimism as resulting from a definitional and a methodological issue. Definitional in that uniqueness was defined as that which lacks comparisons; methodological in that comparisons were understood solely in terms of event-type comparisons. From there we turned to the life sciences, finding there that scientists are motivated to investigate putative uniqueness using heterogeneous tools, models and methods-opportunistically mixing evidence from both event-type and causal-pathway comparisons. We identified two general criteria scientists use to generate such comparisons and importantly, two specific kinds of evolutionary similarity. These allow us to give two non-overlapping characterizations of uniqueness at work in the life sciences. Statistical outliers are trait-bearing lineages at the extreme end of a general trend, while path-dependent cascades are divergent explorations of a lineage through evolutionary space.

This puts us in a position to draw on the case studies and argue against pessimism more forcefully. This we undertake the next section. In the one following, we consider cases of 'apparent uniqueness' before concluding.

\section{Radical pessimism}

Pessimists make a claim about epistemic power and a claim about pursuit: if one targets unique traits, one won't make much epistemic progress and therefore investigations of unique traits will be fruitless. Both claims are mistaken.

According to Tucker, if one lacks event-type comparisons, one cannot generate 'acceptable scientific theories' about the target; for Smith and Wood, a lack of eventtype comparisons makes trying to understand the evolution of our lineage an 'unrealistic research question'. And yet we have seen that analyses of elephant's trunks and human teaching can progress in spite of a lack of event-type comparisons. Explaining human teaching as the outcome of a highly contingent, particular evolutionary cascade does not preclude the development of plausible, well-evidenced hypotheses. Paleoanthropologists lean on causal-pathway comparisons drawn from homologies, 
ethnographic work, the material record and models from cognitive science to generate rich narratives about human teaching. As such, evolutionary uniqueness does not necessarily lead to epistemic pessimism. But this does not mean that optimism should be unbounded.

The optimist blocks the pessimist by appealing to causal-pathway comparisons. But what if causal-pathway comparisons are also impoverished? Jonathan Kaplan (2002) has argued along these lines. He claims that Homo sapiens are too phylogenetically isolated for good inferences about universal, unique traits:

We have no extant relatives which are suspected of sharing similar selective regimes and that can therefore be used to test the fitness consequences of the supposed adaptations. If all or most of the estimated dozen or so extinct hominid species (comprising, perhaps two or three genera) still existed, phylogenetic studies would certainly be easier and might well be useful for distinguishing between competing hypotheses about the spread and maintenance of phenotypic traits of interest. Unfortunately for testing adaptive hypotheses in humans, all the other hominids are extinct and so comparisons between the groups, with special attention to the fitness consequences of differences in key traits, are impossible (Kaplan 2002, 300).

In a nutshell, Kaplan argues that, given the radical uniqueness of hominins, researchers lack access to causal information that would explain the origin and diffusion of salient traits. ${ }^{13}$ But note that Kaplan's argument is restricted to certain kinds of adaptationist hypotheses - one may still be able to answer many other questions concerning human evolution-and that he is restricting himself to certain kinds of token-causal-comparisons, namely, homologous comparisons from other extinct taxa. But it may be that other kinds of proxies can be used to fill in gaps in the story_artefacts and other archaeological remains, say. Moreover, his argument is contingent on future discoveries: indeed, in the decade and a half since his paper, there have been several remarkable finds leading to an increasingly bushy hominin clade (H. floresiensis and the Denisovans, for example, see: Brown 2004; Krause et al. 2010). Finally, pessimism of Kaplan's stripe potentially makes a parallel error to Smith and Wood's. Where the latter ignore causal-pathway comparisons, the former ignores event-type comparisons. Even if one lacks a rich phylogenetic basis for identifying comparisons with our lineages, it does not follow that event-type comparisons are epistemically inert (for instance, see: Emery 2006; Sayers and Lovejoy, 2008; Currie 2013).

From Kaplan's arguments, one can identify an extreme version of uniqueness where both sufficient causal-pathway and event-type comparisons are lacking. In such a circumstance, it is unclear what evidential grounding one might have in understanding our target. Nonetheless we maintain that the inference from putative

\footnotetext{
13 Lewontin has expressed similar pessimism derived from a lack of causal-pathway comparisons: "History and evolution is a form of history, simply does not leave sufficient traces, especially when it is the forces that are at issue. Form and even behavior may leave fossil remains, but forces like Natural Selection do not" $(1988,132)$.
} 
uniqueness to 'unrealistic research questions' is far too quick: it gets the direction of investigation backwards.

Smith and Wood infer from our lineage's uniqueness to pursuit being unjustified. However, as we've seen, establishing the uniqueness or otherwise of elephant's trunks requires a complex array of investigative approaches. Scientists consider several coarse-grained definitions of the trait-is it a proboscis like a tapir, a soft mouth-part like a giraffe's tongue, or a snorkel? — and these definitions are tested in various ways. The process of identifying uniqueness involves stages of empirical investigation. In human teaching, we see both the development of coarse-grained functional definitions of teaching which ground comparative work across taxa, happening in parallel with more human-focused approaches which knit together various strands of causal-pathway evidence.

In short, one cannot know about the uniqueness of a trait independently of an investigation of that trait and so suggesting that putative uniqueness is a reason against pursuit is a mistake. Putative uniqueness is the beginning of a rich set of investigative practices, not a reason to avoid those questions. And indeed, even if the trait in question - be it teaching or trunks - end up being radically unique in our sense, the indirect results of pursuing those explanations are clearly rich. After all, discovering that a trait is indeed unique raises the question of why: what is it about the lineage in question that allowed the trait to emergence and what did other lineages lack?

Thus, both inferring from evolutionary uniqueness to pessimism is unfounded and so is inferring from putative uniqueness to a lack of pursuit-worthiness.

\section{Apparent uniqueness and revisability}

One might object that our account is limited: that it misses attributing uniqueness to cases where it should and attributes uniqueness to cases where it shouldn't.

Consider polyploidy-whole genome duplication-in plants. Polyploidy can occur via a number of routes but tends to bring a common syndrome of effects: plants tend to grow more slowly, produce larger seeds, flower later and live longer. Among angiosperms, there are also often exaggerated changes to the size and shape of reproductive organs as well as increased production of alkaloid chemicals-shifting the relationship between plants and their pollinators and pests (Levin 1983). The effects of polyploidization can be dramatic, should one therefore treat them as unique?

As we've argued, uniqueness attributions are description-dependent and interestrelative. As such, there is latitude to treat any trait as unique and investigate them as such. Yet when the dramatic set of traits described above occurs, we think it will often be more illuminating to situate such traits in the well-understood event-type comparison class of polyploidy. For despite being the outcome of multiple, lowprobability events, polyploidy is a widespread and common event in plant evolution, being particularly common among angiosperms and even more common amongst crop plants. Polyploidy can even be induced in many plants through chemical applications, such as colchicine (Curry 2016). So although polyploidy's effects 
are dramatic - and do not result in the same effects among all species (Soltis et al. 2015)—we think such traits are better situated in well understood event-type generalizations of hybridization, unreduced gamete formation and developmental plasticity. Or to put it another way, the recurrent syndrome of traits associated with polyploidy is not well explored by treating any particular instance as a statistical outlier or a path-dependent cascade.

Contrast this with another evolutionary process that produces striking, singlecase occurrences of traits: the selective survival and extinction of lineage members. Perhaps proboscises evolved multiple times throughout the mammalian clade, but it just so happens that due to unlucky circumstances, only the elephant lineage was successful and persisted over time. Should we attribute uniqueness to the elephant trunk under these circumstances?

For all we know, there are many such cases: lineage extinction is fairly common and the fossil record is extraordinarily patchy. But we would disagree with any attempt that moves from speculative reasoning to a general resistance towards uniqueness attributions. Consider that the case requires that one has knowledge of non-elephantine mammals with trunks, but these mammals left no material traces in the fossil record and indeed, no trace evidence from which current scientists could deduce their existence. Under such circumstance, the question is motivated by an impossible epistemological situation: knowledge of a distribution despite lacking evidence for such knowledge.

Our knowledge of the past is incomplete. But we should not have to rely on omniscience in order to make uniqueness attributions. Instead, what our account makes room for is the revisability of such attributions. Further digging into the fossil record and finer-grained biological surveys may reveal that instances of seemingly unique traits turn out to be more widespread. In fact, we would be surprised if this did not turn out to be the case. Such discoveries would allow researchers to revise (or entrench) their uniqueness attributions.

In short, we don't see either case as undermining our revised understanding of uniqueness attributions. There are striking singulars in the evolutionary record. Some of these will have been the result of systematic, well-understood evolutionary processes like polyploidy. But more generally, singulars point towards the need for epistemic humility - and the need to be open to revising uniqueness attributions in the face of new evidence.

\section{Conclusion}

Life scientists - particularly those interested in hominin evolution-make uniqueness attributions and recognize that these come freighted with epistemic consequences. Nonetheless, with few exceptions, philosophers of science have avoided tackling the notion of uniqueness head on. In this paper, we have shown that an evolutionary account of uniqueness can both make sense of scientists' appeals to the concept and that many have drawn pessimistic conclusions far too quickly. Even if a trait lacks both type-event comparisons and causal-pathways comparisons, finding this out involves a complex set of empirical and theoretical investigations which 
often bear surprising fruit. Uniqueness ought to motivate-not disincentivise- - scientific study.

Acknowledgements The authors are grateful to Rachael Brown, Tim Lewens and Tom Roberts, as well to participants of ISHPSSB 2019, especially Marc Ereshefsky and Matt Haber. Research in this article was supported by grants from the Leverhulme Trust (ECF-2018-005) and the Isaac Newton Trust.

Open Access This article is licensed under a Creative Commons Attribution 4.0 International License, which permits use, sharing, adaptation, distribution and reproduction in any medium or format, as long as you give appropriate credit to the original author(s) and the source, provide a link to the Creative Commons licence, and indicate if changes were made. The images or other third party material in this article are included in the article's Creative Commons licence, unless indicated otherwise in a credit line to the material. If material is not included in the article's Creative Commons licence and your intended use is not permitted by statutory regulation or exceeds the permitted use, you will need to obtain permission directly from the copyright holder. To view a copy of this licence, visit http://creativecommons.org/licen ses/by/4.0\%.

\section{References}

Brigandt I, Love AC (2010) Evolutionary novelty and the evo-devo synthesis: field notes. Evol Biol 37(2-3):93-99

Birch J (2021) Toolmaking and the Origin of Normative Cognition. Biol Philos. 36(4). https://doi.org/10. 1007/s10539-020-09777-9

Boyd R, Richerson PJ (1985) Culture and the Evolutionary Process. University of Chicago Press, Chicago

Brown P, Sutikna T, Morwood MJ, Soejono RP, Saptomo EW, Due RA (2004) A new small-bodied hominin from the Late Pleistocene of Flores. Indonesia Nature 431(7012):1055

Brown RL (2014) What evolvability really is. Br J Philos Sci 65(3):549-572

Caro TM, Hauser MD (1992) Is there teaching in nonhuman animals? Q Rev Biol 67:151-174

Chemero A (2009) Radical Embodied Cognitive Science. MIT Press, Cambridge, MA

Churchland PM (1985) The ontological status of observables: In praise of the superempirical virtues. Images sci. 35-47.

Collingwood RG (1936/1976). Human nature and human history. New York: Ardent Media.

Currie A (2013) Convergence as evidence. Br J Philos Sci 64(4):763-786

Currie A (2018) Rock, bone and ruin: An optimist's guide to the historical sciences. MIT Press, Cambridge, MA

Curry H (2016) Evolution made to order: plant breeding and technological innovation in twentieth-century America. University of Chicago Press, Chicago

Desjardins E (2011a) Reflections on path dependence and irreversibility: lessons from evolutionary biology. Philos Sci 78(5):724-738

Desjardins E (2011b) Historicity and experimental evolution. Biol Philos 26(3):339-364

Dray W (1957) Laws and explanation in history. Oxford University Press, Oxford

Emery NJ (2006) Cognitive ornithology: the evolution of avian intelligence. Philos Trans R Soc Lond B Biol Sci 361:23-43

Ereshefsky M, Turner D (2019) Historicity and explanation. Stud Hist Philos Sci Part A. https://doi.org/10. 1016/j.shpsa.2019.02.002

Gallie WB (1964) Philosophy and the historical understanding. Schocken Books, New York

Gibson J (2015/1986) The ecological approach to visual perception. New York: Psychology Press.

Hannon E, Lewens T (eds) (2018) Why we disagree about human nature. Oxford University Press, Oxford

Hempel CG (1942) The function of general laws in history. J Philos 39(2):35-48

Henrich J (2015) The Secret of our Success: How Culture Is Driving Human Evolution, Domesticating Our Species and Making Us Smarter. Princeton University Press, Princeton

Heyes C (2018) Cognitive Gadgets: The Cultural Evolution of Thinking. Harvard University Press, Cambridge, MA

Hoppitt WJE, Brown GR, Kendal R, Kendal L, Thornton A, Webster MM, Laland KN (2008) Lessons from animal teaching. Trends Ecol Evol 23(9):486-493

Hull DL (1976) Are species really individuals? Syst Zool 25(2):174-191 
Kaplan JM (2002) Historical evidence and human adaptations. Philos Sci 69:S294-S304

Kitcher P (1981) Explanatory unification. Philos Sci 48(4):507-531

Kitcher P (1989) Explanatory unification and the causal structure of the world. In: Explanation S (ed) Philip Kitcher and Wesley C Salmon. University of Minnesota Press, Minneapolis, pp 410-505

Kline MA (2015) How to learn about teaching: An evolutionary framework for the study of teaching behavior in humans and other animals. Behav Brain Sci. e31.

Krause J, Fu Q, Good JM, Viola B, Shunkov MV, Derevianko AP, Pääbo S (2010) The complete mitochondrial DNA genome of an unknown hominin from southern Siberia. Nature 464(7290):894

Kronfeldner M (2018) What's Left of Human Nature? A Post-Essentialist, Pluralist and Interactive Account of a Contested Concept. The MIT Press, Cambridge, MA

Levin DA (1983) Polyploidy and novelty in flowering plants. Am Nat 122(1):1-25

Lewontin RC (1998) The evolution of cognition: Questions we will never answer. In: Scarborough D, Sternberg S (eds) An invitation to cognitive science, Vol 4: Methods, models and conceptual issues. MIT Press, Cambridge, MA, pp 106-132

Losos J (2017) Improbable Destinies: How Predictable is Evolution? Penguin, London

Mackie JL (1965) Causes and Conditions. Am Philos Q 2(4):245-264

McConwell AK (2019) Contingency's causality and structural diversity. Biol Philos 34:26. https://doi.org/10. 1007/s10539-019-9679-x

Milam EL (2019) Creatures of Cain: The Hunt for Human Nature in Cold War America. Princeton University Press, Princeton

Milewski AV, Dierenfeld ES (2013) Structural and functional comparison of the proboscis between tapirs and other extant and extinct vertebrates. Integr Zool 8(1):84-94

Pearce T (2012) Convergence and parallelism in evolution: A Neo-Gouldian account. Br J Philos Sci 63:429-448

Pigliucci M (2008) What, if anything, is an evolutionary novelty? Philos Sci 75(5):887-898

Powell R (2007) Is Convergence more than an analogy? homoplasy and its implications for macroevolutionary predictability. Biol Philos 22:565-578

Powell R (2020) Contingency and convergence: Toward a cosmic biology of body and mind. MIT Press, Cambridge, MA

Premack D (2010) Why humans are unique: three theories. Perspect Psychol Sci 5(1):22-32

Pretorius Y, de Boer WF, Kortekaas K, van Wijngaarden M, Grant RC, Kohi EM, Mwakiwa E, Slotow R, Prins HH (2016) Why elephant have trunks and giraffe long tongues: how plants shape large herbivore mouth morphology. Acta Zoologica 97(2):246-254

Rasmussen LEL, Munger BL (1996) The sensorineural specializations of the trunk tip (finger) of the asian elephant, elephas maximus. Anat Rec 246:127-134

Rietveld E (2012) Bodily intentionality and social affordances in context. In F Paglieri (ed.) Consciousness in Interaction. The role of the natural and social context in shaping consciousness. Amsterdam: John Benjamin.

Sayers K, Lovejoy OC (2008) The chimpanzee has no clothes. Curr Anthropol 49:87-114

Shettleworth SJ (2012) Modularity, comparative cognition and human uniqueness. Philos Trans Royal Soc b: Biol Sci 367:2794-2802

Shoshani J (1998) Understanding proboscidean evolution: a formidable task. Trends Ecol Evol 13(12):480-487

Smith RJ, Wood B (2017) The principles and practice of human evolution research: Are we asking questions that can be answered? CR Palevol 16(5-6):670-679

Soltis PS, Marchant DB, Van de Peer Y, Soltis DE (2015) Polyploid and genome evolution in plants. Curr Opin Genet Dev 35:119-125

Sterelny K (2012) The Evolved Apprentice. The MIT Press, Cambridge, MA

Strevens M (2008) Depth: An Account of Scientific Explanation. Harvard University Press, Cambridge, MA

Thornton A (2008) Variation in contributions to teaching by meerkats. Proceedings Royal Soc b: Biol Sci 275(1644): 1745-1751

Thornton A, McAuliffe K (2006) Teaching in Wild Meerkats. Sci 313(5784):227-229

Tomasello M (2014) A natural history of human thinking. Harvard University Press, Cambridge, MA

Tucker A (1998) Unique events: The underdetermination of explanation. Erkenntnis 48(1):61-83

Tucker A (2011) Historical science, over-and underdetermined: A study of Darwin's inference of origins. Br J Philos Sci 62(4):805-829

Turner DD (2004) The past vs. the tiny: historical science and the abductive arguments for realism. Stud Hist Philos Sci Part A, 35(1), 1-17. 
Van Fraassen BC (1980) The scientific image. Oxford University Press

Vermeij GJ (2006) Historical contingency and the purported uniqueness of evolutionary innovations. Proceedings Nation Al Academy Sci United States of America 103(6):1804-1809

Wagner GP (2014) Homology, genes, and evolutionary innovation. Princeton University Press, Princeton

Walsh D (2015) Organisms, Agency and Evolution. Cambridge University Press, Cambridge

Wong TW (2019) The evolutionary contingency thesis and evolutionary idiosyncrasies. Biol Philos 34(2):22. https://doi.org/10.1007/s10539-019-9684-0

Wong TW (2020) Evolutionary contingency as non-trivial objective probability: Biological evitability and evolutionary trajectories. Stud Hist Philos Sci Part c: Stud Hist Philos Biol Biomed Sci 81:101246. https://doi.org/10.1016/j.shpsc.2019.101246

Publisher's Note Springer Nature remains neutral with regard to jurisdictional claims in published maps and institutional affiliations. 